\title{
Wireless Mobile Communication Using Human Body as a Channel
}

\author{
Mr. Italia Hiren R. ${ }^{1}$, Prof. Dr. G. U. Kharat ${ }^{2}$ \\ ${ }^{1,2}$ (Department of Electronics and Telecommunication Saradchandra Pawar COE, Otur, University of Pune, \\ India)
}

\begin{abstract}
The mobile communication takes place using wired network, infrared and bluetooth which consumes lot of battery power and have security issues. The main objective of research in to mobile communication is to use human body as transmission channel for electrical signals. However, so many experiments has been performed under the research of intra-body communication like capacitive and galvanic coupling to optimize operating frequency, channel length, electrode used etc. In this paper a new methodology has been developed for the alternate wireless mobile communication.
\end{abstract}

Keywords: Wireless Mobile Communication, wireless communication, Mobile to Mobile Communication, Intrabody Communication, IBC HAN.

\section{Introduction}

In today's world transmission of data is performed over a wired network or wireless network. The drawback of the wired network is the routing of the cable. The drawback of the wireless network is packet collision and security risk. But these drawbacks are eliminated by using the proposed system in this paper.

This was originally proposed by T. G. Zimmerman [1]. The concept is to use a human body as communication channel between mobile device terminals. The concept of Personal Area Networks (PAN) [2] Near field IBC is demonstrated how mobile devices near the human body can exchange digital information by capacitive coupling in Pico ampere currents through the human body. In the year 2013 MirHojjat Seyedi [3] et al done a survey to examine the ongoing research in the area of Intra-body communication for body area network applications and gives IBC fundamentals, IBC mathematical models of the human body, IBC transceiver designs. It is found that IBC is a new short range non-RF wireless communication technique specified by the IEEE 802.15 .6 using the human body as a transmission medium. As it stands, the IBC technique potentially provides a more power efficient and naturally secure short range communication method for body area networks, compared to wireless RF. Despite the tremendous benefits, the evolution of IBC is still in its infancy.

Zimmerman [1] said that, the near-field communication can operate at very low frequencies and low transmission power. The prototype of the PAN transmitter operates at $330 \mathrm{kHz}, 30 \mathrm{~V}$, with a transmission power consumption of $1.5 \mathrm{~mW}$ for charging the electrode capacitance. Direct coupling by Masaaki Fukumoto [2] et al is a modified version of the basic capacitive method. The system operates by analog frequency modulation at frequencies within $50 \mathrm{kHz}$ to $90 \mathrm{kHz}$ for transmitting a simple protocol of ID numbers. Sasaki [9] et al tried to illuminate the principles of intra-body communication, where the Electro Optic [EO] sensor is used to receive data signal. Maria Amparo Callej'on [4] et al implemented galvanic [5], [6] and capacitive coupling [7], [8] setups and carried out comprehensive set of measurements by analyzing fundamental IBC parameters such as optimum frequency range, maximum channel length and type of electrodes.

\section{Experimental Setup for the Proposed Model}

The figure 1 shows the experimental setup for the proposed system. At the transmission side the data transferred from Tx pin of RN-42 and received by ARM from Rx of UART1. The data received by ARM is transferred to touch pad using Tx of UART0.

At the reception side the data has been received by touch pad and transmitted to Rx of UART0 of ARM through amplifier LM358. The received ARM data transferred to the RN-42 through Tx of UART1.

Like this way the data transferred from RN-42 to ARM using UART1 and ARM to touch pad by using UART0. The objective of the LM358 is to increase the received data liability. The thing must take care during the experiment about the earthing because the sockets do not have the same ground level. It means the transmitter and receiver have to give same earthing for the proper data transmission. 


\section{Code Overview Future Abstraction}

The figure 2 shows the code overview which is divided into two parts one is Mobile side where GUI elements, BT transmission and BT reception code has been developed in JAVA using Eclipse software. Another is the processor side where BT Tx \& RX and Body Tx \& Rx has been developed for the data transmission in embedded $\mathrm{C}$ using Keil $\mathrm{uVision}$ software. GUI is designed in such a way that it possible to transmit the data and the file which is resides in memory card. The care is also taken care about the security of the transmission it means without the prior permission the data will not get transferred.

Step 1: Send \#

The data has been transfer using the formats given below.

Step 2: Send LI

Step 3: "M" + "Name" + "Mobile" + "Email" + "Address"

Step 4: Send

The file has been received using the formats given below.

Step 1: Send \#

Step 2: Send LI

Step 3: "F" + "File Content"

Step 4: Send

At the time of reception if it receives \# then next two byte has been garbage value and by collecting the next byte it decides whether the information is data or file using " $\mathrm{M}$ " or " $\mathrm{F}$ ". If the information is data then display it on the GUI, and if the information is file then it will store the received file in to the memory card. When it receives the $\sim$ symbol then the data transmission stops.

The figure 3 shows the overall transmission technology. Here the data transfers from bluetooth to ARM and ARM to touch pad at the time of transmission. Here a diode is used to disable the reception of the data by the same transmitter.

\section{Result and Conclusion}

By using this methodology it has been able to transmit the personal data or file which is resides in to the memory card. This transmission may be one-way or two-way depending upon the selection on GUI. So it can be conclude that the data transmission through the human body is possible using the proposed methodology.

\section{Future Abstraction}

Here in this paper we have developed the external hardware to transmit the data from one mobile device to another mobile device. But in the future we can develop the mobile phone with this inbuilt facility. To transmit the data between two devices with the increase data rate and increased buffered size. This may also be expands to transmit the audio, video or image file. By installing this application in the printer, by touching the printer simultaneously with device we will be able to print the document. By handshaking we will be able to transmit the data between two devices which is carried by the humans. If we connect the all PCs on the conference table then we will be able to transmit the data over all the PCs at the same time.

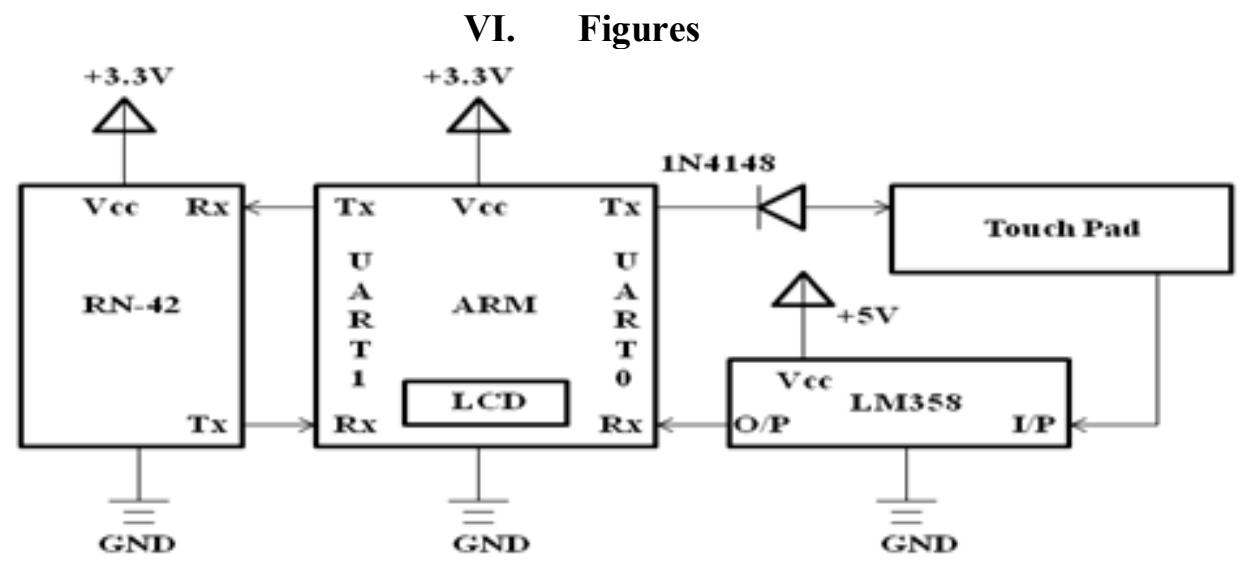

Fig. 1. Experimental Setup of Proposed System 


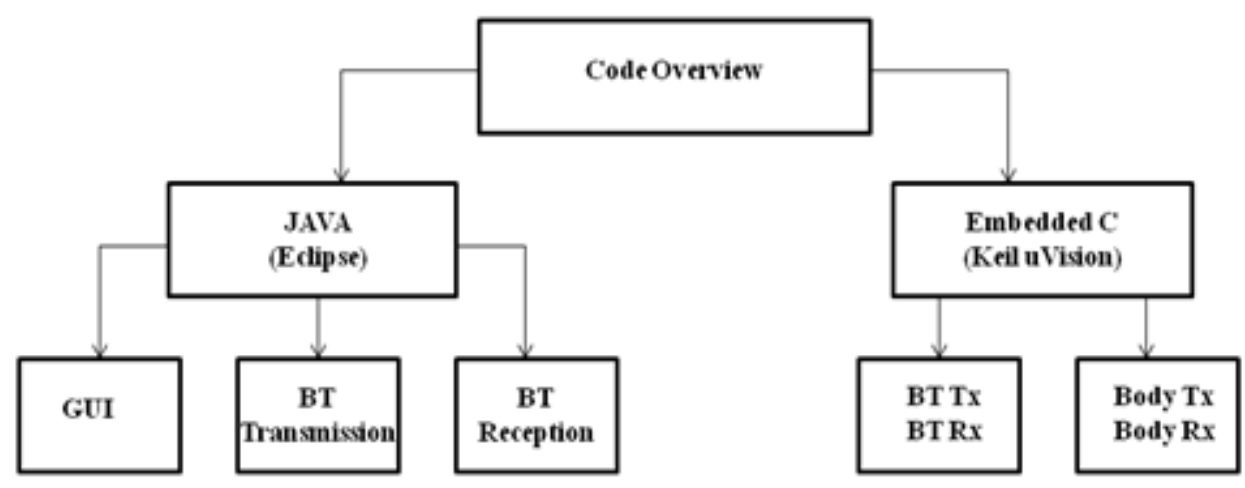

Fig. 2. Code Overview

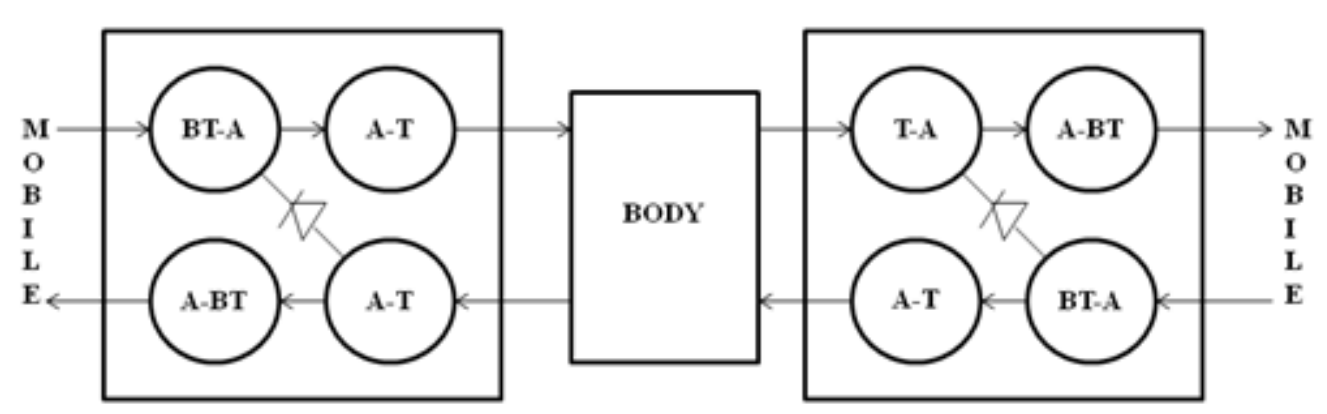

Fig. 3. Transmission Technology

\section{References}

[1] T. G. Zimmerman, "Personal area network (PAN)," M.S. thesis, Media Lab., Massachusetts Inst. Technol., Cambridge, Mar. 1995.

[2] Mitsuru Shinagawa, Masaaki Fukumoto, Katsuyuki Ochiai, and Hakaru Kyuragi, "A Near-Field-Sensing Transceiver for Intrabody Communication Based on the Electro optic Effect," IEEE Transactions On Instrumentation And Measurement, vol. 53, no. 6, pp. 1533-1538, Dec. 2004.

[3] MirHojjat Seyedi,Behailu Kibret, Daniel T. H. Lai, and Michael Faulkner, Member, IEEE, "A Survey on Intrabody Communications for Body Area Network Applications," IEEE Transactions On Biomedical Engineering, vol. 60, no. 8, pp. 20672079, Aug. 2013.

[4] Maria Amparo Callej'on, David Naranjo-Hern'andez, Javier Reina-Tosina, and Laura M. Roa, "A Comprehensive Study into Intrabody Communication Measurements," IEEE Transactions On Instrumentation And Measurement, vol. 62, no. 9, pp. 2446-2455 Sep. 2013.

[5] M. S. Wegmueller, A. Kuhn, J. Froehlich, M. Oberle, N. Felber, N. Kuster, and W. Fichtner, “ An attemp to model the human body as a communication channel," IEEE Transactions On Instrumentation And Measurement, vol. 54, no. 10, pp. 1851-1857, Oct. 2007.

[6] Y. Song, Q. Hao, K. Zhang, M. Wang, Y. Chu, and B. Kang, “ The simmulation method of the galvanic intrabody communication with different signal transmission path," IEEE Transactions On Instrumentation And Measurement, vol. 60, no. 4, pp. 1257-1266, Apr. 2011.

[7] R. Xu, H. Zhu, and J. Yuan, "Electric-field intrabody communication channel modelling with finite element method," IEEE Transactions on Instrumentation and Measurement, vol. 58, no. 3, pp. 705-712, Mar. 2011.

[8] Z. Lucev, I. Krois, and M. Cifrek, "A capacitive intrabody communication channel from $100 \mathrm{kHz}$ to $100 \mathrm{MHz}$," in Proc. IEEE Instrum. Meas. Technol. Conf., May 2011, pp. 1-4.

[9] Ai-ichiro Sasaki, Mitsuru Shinagawa, Katsuyuki Ochiani, "Principles and Demostrastion of intrabody communication with a sensitive electro optic sensor," IEEE Transactions on Instrumentation and Measurement, vol. 58, no. 2, pp. 457-466, Feb. 2009.

[10] Brian W. Kernighan, Dennis M. Ritchie, The C programming Language, First Edition 1988, Prentice-Hall, ISBN 0-13-110370-9.

[11] http://www.cadsoftusa.coom/download-eagle/

[11] Ramakant A. Gayakwad, Op-Amps and Linear Integrated Circuits, 4thEdition, Prentice-Hall.

[12] Robert L. Boylestad, Louis Nashelsky, Electronic Devices and Circuit Theory, 10thEdition, Prentice-Hall. 